\title{
Best Research Practice Implementation: The Experience of the N.N. Burdenko National Medical Research Center of Neurosurgery
}

\author{
Gleb DANILOV ${ }^{\mathrm{a}, 1}$, Michael SHIFRIN ${ }^{\mathrm{a}}$, Yulia STRUNINA ${ }^{\mathrm{a}}$, Timur ISHANKULOV ${ }^{\mathrm{a}}$, \\ Timur ZAGIDULLIN ${ }^{\mathrm{a}}$, Elizaveta MAKASHOVA ${ }^{\mathrm{a}}$, Igor PRONIN ${ }^{\mathrm{a}}$, \\ Nikolay KONOVALOV ${ }^{\mathrm{a}}$ and Alexander POTAPOV ${ }^{\mathrm{a}}$ \\ ${ }^{a}$ Laboratory of Biomedical Informatics and Artificial Intelligence, National Medical \\ Research Center of Neurosurgery named after N.N. Burdenko, Moscow, Russian \\ Federation
}

\begin{abstract}
Implementing the best research principles initiates an important shift in clinical research culture, improving efficiency and the level of evidence obtained. In this article, we share our own view on the best research practice and our experience introducing it into the scientific activities of the N.N. Burdenko National Medical Research Center of Neurosurgery (Moscow, Russian Federation). While being adherent to the principles described in the article, the percentage of publications in the international scientific journals in our Center has increased from $7 \%$ to $27 \%$, with an overall gain in the number of articles by 2 times since 2014 . We believe it is important that medical informatics professionals equally to medical experts involved in clinical research are familiar with the best research principles.
\end{abstract}

Keywords. Best research practice, neurosurgery, data management, FAIR, biostatistics

\section{Introduction}

Medicine is traditionally an area entirely focused on practice and application: providing the most effective, safe, and cost-effective care for people with health issues. Probably due to the complexity of human biology and our limited knowledge of it, medicine is difficult to formalize. Many concepts do not have clear and generally accepted definitions. Many definitions are superficial and rely only on the explicit pathology manifestation. At the same time, the concept of evidence-based medicine, which has gained a deserved popularity and recognition in recent decades, postulates scientific verification of the effectiveness, safety, and economic feasibility of medical interventions as the basis for decision-making in medicine [2]. Scientific hypothesis testing inevitably relies on formalization, logic, and rigorous operational definitions, essential for exact sciences. Due to the specificity of training and practice in medicine, these approaches are not at the forefront in the system of a doctor's professional thinking. The culture of well-designed research is not taken for granted in medicine. The research activity itself requires serious multidisciplinary support, in which the role of medical

\footnotetext{
${ }^{1}$ Corresponding author, Gleb Danilov, the N.N. Burdenko National Medical Center of Neurosurgery, 4th Tverskaya-Yamskaya str. 16, Moscow 125047, Russian Federation; E-mail: glebda@yandex.ru.
} 
informatics is of vital importance. The quality of research outcome cannot rest solely with doctors. IT specialists are much responsible for handling data, which necessitates a good understanding of clinical research ethics, regulation, and methodology.

The pharmaceutical industry has developed best practices in human research over the years. We have to admit that research opportunities tend to be more restrained in the academic field than in big pharma. However, in our opinion, implementing best research principles is a cultural shift that requires not so much money as changing the way of thinking. In this article, we want to share our own experience of introducing the best research practices into scientific activities of the N.N. Burdenko National Medical Research Center of Neurosurgery (the NSC, former the N.N. Burdenko Neurosurgery Institute, Moscow, Russian Federation).

\section{Rationale for best research practice implementation in medical research}

The NSC is a leading neurosurgical institution in the Russian Federation, one of the largest neurosurgical facilities in the world. The National Neurosurgery Center has 300 beds and annually performs up to 10,000 neurosurgical interventions with a postoperative mortality rate of less than $0.5 \%$. For 21 years of medical information systems operation, the National Center for Neurosurgery has accumulated unique and large data archives, mining of which is absolutely justified but not easy. The data generation speed in high-tech neurosurgery exceeds the ability to extract knowledge from data. Thus, the strategic goals for the development of the NSC research activities are:

- to improve the level of research quality and evidence due to best research practices

- to increase the efficiency of the secondary data use in scientific research

- to improve data quality being collected in research

- to increase the likelihood of papers acceptance in high-ranked journals

- to increase the number of citations

To achieve these goals, it seems appropriate to influence the complex research process at its consecutive stages.

\section{Key research principles we consider}

Below we list the main ideas we concentrated on to improve our research practice. These are research planning, biostatistics, data management, reproducible statistical analysis, medical writing and project management. All the research principles we enlist follow the good clinical practice (GCP) statement, an international ethical and scientific quality standard for designing, recording, and reporting research with human subjects involvement. We consider sticking to this standard obligatory. That is why we believe the experts in medical informatics should invest a certain time to get acquainted with the ICH GCP document [4]. 


\subsection{Research planning}

Good research practice starts with resolving ethics and legal issues. Is it legal and moral to do a certain study in humans? Does it carry more benefits than risks for patients? Is it possible and necessary to protect or insure the participants from unfavorable events related to a study? One should answer all these questions before the study starts taking into account national and global regulations.

The technical part of research begins with a well-thought plan written normally in the form of a protocol. Writing a rigorous research plan sounds like a well-known principle, nevertheless commonly underestimated and even regularly ignored. Writing a research protocol could be intently addressed in a series of articles - this is such an important principle. It completely determines all subsequent stages of the research process and, therefore, the success in achieving the results, the likelihood of publication, and any other "return on investment." Planning a study provides an unambiguous goal statement, helps minimize common errors and biases in research aim, design choice, patient selection, data collection, data analysis, interpretation and presentation of results, etc.

At the NSC we developed a protocol template which includes the following sections:

- $\quad$ title page with main project identifiers,

- project team,

- definitions and abbreviations,

- $\quad$ project goals and tasks,

- population with inclusion/exclusion criteria,

- rationale/background for the study,

- research design and methodology with project scheme,

- randomization plan (when necessary),

- $\quad$ primary and secondary end points,

- data management plan,

- $\quad$ statistical analysis plan,

- $\quad$ case report form (CRF),

- additional resources,

- informed consent,

- investigator commitments,

- local advisory board approvement,

- local ethics board approvement,

- references.

We consider filling in these sections is necessary to ensure the best research practice in the upcoming research procedures as requires by GCP statement.

Certain mnemonic rules facilitate the start of writing a protocol (such as PICO Patient, Intervention, Comparator, Outcome). We believe in doing the following first when planning a project:

- Summarizing known (via systematic literature search)

- Defining unknown

- Asking a proper research question

- Defining the right research hypothesis

The goal statement, design, data collection scheme come accordingly to the main hypothesis. 
This principle is overrated and underestimated simultaneously. Medical experts often believe that the fact of statistical analysis itself determines the study's success. Yet, statistical analysis is secondary to the correct task formulation and the quality of the collected data. Biostatistician participates in study planning and writing a research protocol, translates the main hypothesis into a formalized version which is testable with a statistical approach, chooses research design, defines the set of data to collect, picks up the statistical tests, calculates sample sizes to guarantee research power, performs data analysis. It is quite clear that without biostatistics, evidence-based medicine has no foundation. A biostatistician is a trained specialist a doctor cannot fully replace, even if the latter has mastered certain statistical analysis methods. We believe it is important to involve such a specialist in a clinical research team.

\subsection{Data management}

Data management is a key process aimed to ensure the unambiguity, completeness, security, and reliability of data collection and storage in research. The efficiency and quality of the data management are assured with adherence to GCP using clinical data management systems (CDMS). At the NSC, a stand-alone version of the CDMS REDCap is used as a data management tool in studies planned since 2017 [1,3]. This system provides data entry organization in electronic CRF, a unified structure, format, integrity, security, availability, verification, and quality control of data, which is important for the reproducibility of statistical analysis, information security, and multicenter distributed research, final research quality. In fact, the system contributes to the implementation of the FAIR principles into clinical research [5].

\subsection{Reproducible statistical analysis}

The statistical analysis becomes reproducible with code written in programming languages. Compared with push-button interface in statistical software, scripts are beneficial in reproducibility when typical data analysis must be repeated many times as data accumulates. Reporting the analysis procedure in code is another quality control tool. At the NSC, we typically use R for common statistical analysis and normally code in Python for machine learning projects.

\subsection{Medical writing}

Comments on the quality of scientific English text written by non-native speakers are typical from journal reviewers and editors. However, writing a text in proper English is only part of the task. Complete and clear reporting of research results is no less important for their subsequent correct interpretation. The EQUATOR (Enhancing the QUAlity and Transparency Of health Research) Network is an international initiative to improve the reliability and value of published health research literature by promoting transparent and accurate reporting and wider use of robust reporting guidelines [6]. Nowadays, many medical journals require following these guidelines when submitting a manuscript. However, we strongly advise adopting them in every research report as a best practice. 


\subsection{Project management}

No task can be accomplished without control and sometimes pushing. Even the most well-planned research can come to naught without good management, especially when many stakeholders are involved. The "research orchestra" conductor is a project manager who understands when different activities must be completed and controls the overall movement towards the goal.

\section{The results of best practice implementation}

Shifting towards a new research culture with adherence to the above-mentioned principles has influenced the productivity of scientific research in our Center in recent years. Since 2017, data for almost 60 scientific projects have been managed in REDCap. Since 2018, research protocols are developed for almost all new projects. Although this research paradigm is instilled gradually and not all principles are well perceived by medical experts, the percentage of publications in the international peer-reviewed scientific journals has increased from $7 \%$ ( 8 of 111 ) to $27 \%$ (60 of 222 ), with an overall gain in the number of scientific articles by 2 times since 2014 .

\section{Conclusion}

High-quality research, regardless of its objects and subjects, can be ensured by adhering to a set of principles and engaging a multidisciplinary team with dedicated competencies in research planning, data collection and analysis, and project management. We believe it is important that medical informatics professionals equally to medical experts involved in clinical research are familiar with these principles.

Our work was supported by Russian Foundation for Basic Research (grant 18-2901052).

\section{References}

[1] Danilov GV, Shifrin MA, Strunina YV, Pronkina TE, Ishankulov TA, Burov AA, Dorofeyuk YA, and Potapov AA. Clinical Research Data Management: Experience of the N.N. Burdenko NMRC of Neurosurgery [in Russian]. Vrach i Inf. Tehnol. 2020; 6-14.

[2] Djulbegovic B, Guyatt GH. Progress in evidence-based medicine: a quarter century on. The Lancet. 2017 Jul 22;390(10092):415-23.

[3] Harris PA, Taylor R, Minor BL, Elliott V, Fernandez M, O'Neal L, McLeod L, Delacqua G, Delacqua F, Kirby J, Duda SN. The REDCap consortium: Building an international community of software platform partners. Journal of biomedical informatics. 2019 Jul 1;95:103208.

[4] INTERNATIONAL COUNCIL FOR HARMONISATION OF TECHNICAL REQUIREMENTS FOR PHARMACEUTICALS FOR HUMAN USE (ICH) ICH HARMONISED GUIDELINE INTEGRATED ADDENDUM TO ICH E6(R1): GUIDELINE FOR GOOD CLINICAL PRACTICE E6(R2), (2016). https://database.ich.org/sites/default/files/E6_R2_Addendum.pdf (accessed July 30, 2021).

[5] FAIR Principles - GO FAIR, (n.d.). https://www.go-fair.org/fair-principles/ (accessed July 30, 2021).

[6] The EQUATOR Network | Enhancing the QUAlity and Transparency Of Health Research, (n.d.). https://www.equator-network.org/ (accessed July 30, 2021). 